\title{
Sums and products of intervals in ordered groups and fields
}

\section{Tamás Glavosits}

University of Miskolc,

Department of Applied Mathematics, Miskolc-Egyetemváros, Hungary, H-3515 email: matgt@uni-miskolc.hu

\section{Zsolt Karácsony \\ University of Miskolc,} Department of Applied Mathematics, Miskolc-Egyetemváros, Hungary, H-3515 email: matkzs@uni-miskolc.hu

\begin{abstract}
We show that the sum of two intervals in an ordered dense Abelian group is also an interval such that the endpoints of the sum are equal to the sums of the endpoints. We prove analogous statements concerning to the product of two intervals.
\end{abstract}

\section{Introduction}

It is well known from elementary real analysis that if $a, b, c, d$ are real numbers with $\mathrm{a}<\mathrm{b}$ and $\mathrm{c}<\mathrm{d}$, then

$$
] \mathrm{a}, \mathrm{b}[+] \mathrm{c}, \mathrm{d}[=] \mathrm{a}+\mathrm{c}, \mathrm{b}+\mathrm{d}[,
$$

moreover, if $0 \leqslant \mathrm{a}<\mathrm{b}$ and $0 \leqslant \mathrm{c}<\mathrm{d}$, then

$$
] \mathrm{a}, \mathrm{b}[\cdot] \mathrm{c}, \mathrm{d}[=] \mathrm{ac}, \mathrm{bd}[.
$$

The main purpose of this article is to show that equations (1), (2) remain valid in more general settings. Our references to ordered structures are [4], [8], [12], [13], [18].

2010 Mathematics Subject Classification: 39B22

Key words and phrases: interval, ordered dense Abelian gropup, ordered field, interval arithmetic, interval calculus, interval analysis 
Now, we shall give a sort list of the necessary concepts and notations:

We say that $X=X(\leqslant)$ is a partially ordered set or a poset if $X$ is a set and $\leqslant$ is a relation on $X$ such that it is reflexive, symmetric and transitive.

A poset $X=X(\leqslant)$ is said to be ordered or a loset, if either $x \leqslant y$ or $y \leqslant x$ for all $x, y \in X$.

Let $X=X(*)$ be a groupoid in the sense that $X$ is a nonempty set, $*$ is a binary operation on $X$. Then for any $A, B \subseteq X$ and $a \in X$ define

$$
\begin{aligned}
& A * B:=\{a * b \in X \mid a \in A, b \in B\}, \\
& a * B:=\{a\} * B .
\end{aligned}
$$

Let $X=X(\leqslant)$ be a poset and $a, b \in X$ such that $a<b$, that is, $a \leqslant b$ but $a \neq b$. The open interval is defined by

$$
] \mathrm{a}, \mathrm{b}[:=\{x \in X \mid \mathrm{a}<\mathrm{x} \text { and } \mathrm{x}<\mathrm{b}\} .
$$

The $a$ and $b$ are the endpoints of the interval $] a, b[$. Similarly, we can define ] $\mathrm{a}, \mathrm{b}]:=\{x \in X \mid \mathrm{a}<\mathrm{x} \leqslant \mathrm{b}\},[\mathrm{a}, \mathrm{b}[:=\{x \in X \mid \mathrm{a} \leqslant \mathrm{x}<\mathrm{b}\},[\mathrm{a}, \mathrm{b}]:=\{x \in X \mid$ $a \leqslant x \leqslant b\}$.

A poset $X=X(\leqslant)$ is said to be dense (in itself) if $] x, y[\neq \emptyset$ for all $x, y \in X$ with $x<y$.

An ordered group $\mathbb{G}=\mathbb{G}(+, \leqslant)$ is a group together with an order that is compatible with the group operation. A set of all positive elements of an ordered group $\mathbb{G}$ is denoted by $\mathbb{G}_{+}$, that is, $\mathbb{G}_{+}:=\{x \in \mathbb{G} \mid x>0\}$.

An ordered group $\mathbb{G}=\mathbb{G}(+, \leqslant)$ is said to be Archimedean ordered if for all $x, y \in \mathbb{G}_{+}$there exits a positive integer $n$ such that $n x:=x+\cdots+x>y$.

An ordered field $\mathbb{F}=\mathbb{F}(+, \cdot, \leqslant)$ is a field (the operation $\cdot$ is commutative) together with an order that is compatible with the field operations, in the sense, that if $x \leqslant y$, then $x+z \leqslant y+z$ for all $x, y, z \in \mathbb{F}$ and if $x \leqslant y$, then $x z \leqslant y z$ for all $x, y \in \mathbb{F}$ and $z \in \mathbb{F}_{+}:=\{x \in \mathbb{F} \mid x>0\}$.

The foundations of the so-called interval arithmetic were laid by E. Moore, the first appearance of this topic was in 1959 [14], see also [15], [16] and [1]. Now, we shall show the Moore's formulas

$$
\begin{aligned}
{[\underline{a}, \bar{a}]+[\underline{b}, \bar{b}] } & =[\underline{a}+\underline{b}, \bar{a}+\bar{b}], \\
{[\underline{a}, \bar{a}]-[\underline{b}, \bar{b}] } & =[\underline{a}-\bar{b}, \bar{a}-\underline{b}], \\
{[\underline{a}, \bar{a}] \cdot[\underline{b}, \bar{b}] } & =[\min (\underline{a b}, \underline{a} \bar{b}, \bar{a} \underline{b}, \overline{a b}), \max (\underline{a b}, \underline{a} \bar{b}, \bar{a} \underline{b}, \overline{a b})], \\
{[\underline{a}, \bar{a}] /[\underline{b}, \bar{b}] } & =[\underline{a}, \bar{a}] \cdot[1 / \bar{b}, 1 / \underline{b}] \quad 0 \notin[\underline{b}, \bar{b}]
\end{aligned}
$$

for all $\underline{a}, \bar{a}, \underline{b}, \bar{b} \in \mathbb{R}$ with $\underline{a} \leqslant \bar{a}$ and $\underline{b} \leqslant \bar{b}$. 
Due to [9] the results of Moore was extended to open ended unbounded intervals by R. J. Hanson (1968) [5], W. Kahan (1968) [10], E. Davis (1987) $[2]$.

The famous Kohan-Novoa-Ratz arithmetic concerning to the division by an interval coutaining zero can be found in [11]:

$$
\mathbf{a} / \mathbf{b}=\left\{\begin{array}{r}
\mathbf{a} \cdot[1 / \bar{b}, 1 / \underline{b}] \text { for } 0 \notin \mathbf{b} \\
{[-\infty,+\infty] \text { for } 0 \in \mathbf{a} \text { and } 0 \in \mathbf{b}} \\
{[\overline{\mathbf{a}} / \underline{\mathrm{b}},+\infty] \text { for } \overline{\mathrm{a}}<0 \text { and } \underline{\mathrm{b}}<\overline{\mathrm{b}}=\underline{0}} \\
{[-\infty, \overline{\mathrm{a}} / \overline{\mathrm{b}}] \cup[\overline{\mathrm{a}} / \underline{\mathrm{b}},+\infty] \text { for } \overline{\mathrm{a}}<0 \text { and } \underline{\mathrm{b}}<0<\overline{\mathrm{b}}} \\
{[-\infty, \overline{\mathrm{a}} / \overline{\mathrm{b}}] \text { for } \overline{\mathrm{a}}<0 \text { and } 0=\underline{\mathrm{b}}<\overline{\mathrm{b}}} \\
{[-\infty, \underline{\mathrm{a}} / \underline{\mathrm{b}}] \text { for } 0<\underline{\mathrm{a}} \text { and } \underline{\mathrm{b}}<\overline{\mathrm{b}}=\underline{0}} \\
{[-\infty, \underline{\mathrm{a}} / \underline{\mathrm{b}}] \cup[\underline{\mathrm{a}} / \underline{\mathrm{b}},+\infty] \text { for } 0<\underline{\mathrm{a}} \text { and } \underline{\mathrm{b}}<0<\overline{\mathrm{b}}} \\
{[\underline{\mathrm{a}} / \overline{\mathrm{b}},+\infty] \text { for } \underline{\mathrm{a}}<0 \text { and } 0=\underline{b}<\overline{\mathrm{b}}} \\
\emptyset \text { for } 0 \notin \mathbf{a} \text { and } 0 \in \mathbf{b}
\end{array}\right.
$$

for all closed ended bounded interval $\mathbf{a}, \mathbf{b}$ of the real line.

An other way to extend the results of E. Moore that is to use the set $\overline{\mathbb{R}}=$ $\mathbb{R} \cup\{-\infty,+\infty\}$ with uppear additions of J. Moreau [17], that is,

$$
+\infty+(+\infty)=+\infty \text { and }+\infty+(-\infty)=+\infty .
$$

For example, in [3] can be found that

$$
\begin{array}{ll}
] x+y,+\infty[=] x, \infty]+] y,+\infty[ & (x, y \in \overline{\mathbb{R}}), \\
] x+y,+\infty]=[x, \infty]+[y,+\infty] & (x, y \in \overline{\mathbb{R}}), \\
{[-\infty, x+y[=[-\infty, x[+]-\infty, y]} & (x, y \in \mathbb{R} \cup\{-\infty\} \text { or } \mathbb{R} \cup\{+\infty\}), \\
{[-\infty, x+y]=[-\infty, x]+[-\infty, y]} & (x, y \in \mathbb{R} \cup\{-\infty\} \text { or } \mathbb{R} \cup\{+\infty\})
\end{array}
$$

In $[11]$ the author use intervals $X=[\underline{X}, \bar{X}]$ where $\underline{X}$ is the vector or matrix whose components are lower bounds of corresponding components of $\mathrm{X}$, and $\overline{\mathrm{X}}$ is the vector or matrix whose components are upper bounds of corresponding components of X.

In our former paper [6] we have investigated the sums and the products of intervals in ordered semigroups.

In our present paper we investigate the sums of open ended bounded intervals in ordered groups and the products of open ended buonded intervals in 
ordered fields. To obtained results will be used to extend additive and logarithmic functions [7].

Cases that arise when proving results for a product of two intervals a and b can be grouped according to the following criteria: the point 0 is an interior point neither of $\mathbf{a}$ nor of $\mathbf{b}$; the point 0 is an interior point either of $\mathbf{a}$ or of $\mathbf{b}$; the point 0 is an interior point both of $\mathbf{a}$ and $\mathbf{b}$.

Finally, it is worth mentioning that if $X=X(\leqslant)$ is a loset, $] a, b[\subseteq X$ and $c \in] a, b[$, then

$$
] \mathrm{a}, \mathrm{b}[=] \mathrm{a}, \mathrm{c}] \cup] \mathrm{c}, \mathrm{b}[.
$$

If $X=X(\leqslant)$ is only a poset but is not a loset, then $(5)$, in general, is not true. Thus, our applied arguments lose their validity on ordered structures in which the order is not linear.

\section{Sum of intervals in ordered dense Abelian groups}

In this section $\mathbb{G}=\mathbb{G}(+, \leqslant)$ is an ordered dense Abelian group, $\underline{\mathrm{a}}, \overline{\mathrm{a}}, \underline{\mathrm{b}}, \overline{\mathrm{b}}$ $\gamma \in \mathbb{G}$ with $\underline{a}<\overline{\mathrm{a}}$ and $\underline{\mathrm{b}}<\overline{\mathrm{b}}$.

The following Proposition is trivial.

Proposition $1 \gamma+] \underline{\mathrm{a}}, \overline{\mathrm{a}}[=] \gamma+\underline{\mathrm{a}}, \gamma+\overline{\mathrm{a}}[$, and $\gamma+] \underline{\mathrm{a}}, \overline{\mathrm{a}}]=] \gamma+\underline{\mathrm{a}}, \gamma+\overline{\mathrm{a}}]$.

Proposition 2 If $\alpha, \beta \in \mathbb{G}_{+}$, then $] 0, \alpha+\beta[\subseteq] 0, \alpha[+] 0, \beta[$.

Proof. Let $x \in] 0, \alpha+\beta[$. Since $0<\alpha<\alpha+\beta$ there are two cases:

1 . Assume that $x \in] 0, \alpha$. Since $x>0$ and $\beta>0$ there exists an $y \in \mathbb{G}_{+}$such that $y<x$ and $y<\beta$. Since $y<x<x+y$ thus we have that $0<x-y<x \leqslant \alpha$ whence we obtain that

$$
x=(x-y)+y \in] 0, \alpha[+] 0, \beta[.
$$

2. Assume that $x \in] \alpha, \alpha+\beta[$. Then by Proposition 1. $x-\alpha \in] 0, \beta[$ thus there exists an element $y \in \mathbb{G}_{+}$such that $y<\beta-(x-\alpha)$ and $y<\alpha$. Since $y<\alpha<\alpha+y$ thus we have that $0<\alpha-y<\alpha$. Since $y<\beta-(x-\alpha)$ thus we have that $0<x-\alpha<y+(x-\alpha)=x+y-\alpha<\beta$. Thus

$$
x=(\alpha-y)+(x+y-\alpha) \in] 0, \alpha[+] 0, \beta[.
$$

The following Proposition is trivial. 
Proposition 3$] \underline{a}, \bar{a}[+] \underline{b}, \bar{b}[\subseteq] \underline{a}+\underline{b}, \bar{a}+\bar{b}[$.

Theorem 1$] \mathrm{a}, \overline{\mathrm{a}}[+] \underline{\mathrm{b}}, \overline{\mathrm{b}}[=] \overline{\mathrm{a}}+\underline{\mathrm{b}}, \overline{\mathrm{a}}+\overline{\mathrm{b}}[$.

Proof. By Proposition 3 it is enough to show that

$$
] \underline{a}+\underline{b}, \bar{a}+\bar{b}[\subseteq] \underline{a}, \bar{a}[+] \underline{b}, \bar{b}[\text {. }
$$

By Proposition 1. and by Proposition 2. we have that

$$
\begin{aligned}
] \underline{a}+\underline{b}, \bar{a}+\bar{b}[= & (\underline{a}+\underline{b})+] 0,(\bar{a}-\underline{a})+(\bar{b}-\underline{b})[\subseteq \\
& (\underline{a}+] 0, \bar{a}-\underline{a}[)+(\underline{b}+] 0, \bar{b}-\underline{b}[)= \\
& ] \underline{a}, \bar{a}[+] \underline{b}, \bar{b}[
\end{aligned}
$$

The following Theorem can be easily obtained by simply calculation.

Theorem 2 If $\mathbb{G}=\mathbb{G}(+, \cdot, \leqslant)$ is an Archimedean ordered group, then the following assertions are equivalent:

1. $\mathbb{G}$ is dense.

2. ] $\underline{a}, \bar{a}[+] \underline{b}, \bar{b}[=] \underline{a}+\underline{b}, \bar{a}+\bar{b}[$ for all $\underline{a}, \bar{a}, \underline{b}, \bar{b} \in \mathbb{G}$ with $\underline{a}<\bar{a}$ and $\underline{b}<\bar{b}$.

3. $\mathbb{G}(+, \leqslant)$ is not isomorphic to the ordered group $\mathbb{Z}=\mathbb{Z}(+, \leqslant)$ (which is the group of all integers).

\section{The products of intervals in ordered fields}

In this section $\mathbb{F}=\mathbb{F}(+, \cdot, \leqslant)$ is an ordered field, $\underline{a}, \bar{a}, \underline{b}, \bar{b} \in \mathbb{F}$ with $\underline{a}<\bar{a}$ and $\underline{\mathbf{b}}<\overline{\mathbf{b}}$. Define the intervals $\mathbf{a}$ and $\mathbf{b}$ by

$$
\mathbf{a}:=] \underline{\mathbf{a}}, \overline{\mathrm{a}}[\quad \text { and } \quad \mathbf{b}:=] \underline{\mathrm{b}}, \overline{\mathrm{b}}[\text {. }
$$

As a temporary device, use the notation for any open ended bounded interval $\mathbf{x}$ that

$$
\begin{aligned}
& 0<\mathbf{x}, \text { if } 0<x \text { for all } x \in \mathbf{x}, \\
& \mathbf{x}<0, \text { if } x<0 \text { for all } x \in \mathbf{x} .
\end{aligned}
$$

Proposition 4 If $\alpha, \beta, \gamma \in \mathbb{F}$ with $\alpha<\beta$, then

$$
\gamma \cdot] \alpha, \beta\left[= \begin{cases}] \gamma \beta, \gamma \alpha[, & \text { if } \gamma<0 \\ ] \gamma \alpha, \gamma \beta[, & \text { if } \gamma>0 .\end{cases}\right.
$$


Proof. If $\gamma>0$ and $x \in] \gamma \alpha, \gamma \beta\left[\right.$, then $\gamma \alpha<x<\gamma \beta$ thus $\alpha<\frac{x}{\gamma}<\beta$ whence

$$
\left.x=\gamma \cdot \frac{x}{\gamma} \in \gamma \cdot\right] \alpha, \beta[.
$$

The converse inclusion is trivial.

The case $\gamma<0$ can be proved analogously.

The following Proposition is trivial.

Proposition 5 If $0<\mathbf{a}$ and $0<\mathbf{b}$, then $\mathbf{a} \cdot \mathbf{b} \subseteq] \underline{a b}, \bar{a} \bar{b}[$.

Proposition 6 If $0<\mathbf{a}$ and $0<\mathbf{b}$, then $] \underline{\mathrm{ab}}, \overline{\mathrm{a}} \overline{\mathrm{b}}[\subseteq \mathbf{a} \cdot \mathbf{b}$.

Proof. Let $x \in] \underline{a b}, \bar{a} \bar{b}[$. There are two cases:

1. If either $\underline{a}=0$ or $\underline{b}=0$, say $\underline{a}=0$, then there exists an $\varepsilon>0$ such that

$$
\varepsilon<\overline{\mathrm{b}}-\frac{\mathrm{x}}{\overline{\mathrm{a}}}, \quad \text { and } \quad \varepsilon<\overline{\mathrm{b}}-\underline{\mathrm{b}} \text {. }
$$

Since $0<\varepsilon<\bar{b}-\frac{x}{\bar{a}}$ we have that $0<\frac{x}{\bar{b}-\varepsilon}<\bar{a}$. Since $0<\varepsilon<\bar{b}-\underline{b}$ we have that $\underline{b}<\bar{b}-\varepsilon<\bar{b}$. Thus we obtain that

$$
\left.x=\frac{x}{\bar{b}-\varepsilon} \cdot(\bar{b}-\varepsilon) \in\right] \underline{a}, \bar{a}[\cdot] \underline{b}, \bar{b}[.
$$

2. If $\underline{a} \neq 0$ and $\underline{b} \neq 0$, then it is easy to see that $\underline{a b}<\underline{a} \bar{b}<\bar{a} \bar{b}$ thus there is two sub-cases:

a. If $x \in] \underline{a b}, \underline{a} \bar{b}]$, then there exists an $\varepsilon>0$ such that

$$
\varepsilon<\overline{\mathrm{a}}-\underline{\mathrm{a}} \text { and } \varepsilon<\frac{x}{\underline{\mathrm{b}}}-\underline{\mathrm{a}} .
$$

Since $0<\varepsilon<\overline{\mathrm{a}}-\underline{\mathrm{a}}$ thus we have that $\underline{\mathrm{a}}<\underline{\mathrm{a}}+\varepsilon<\overline{\mathrm{a}}$ and since $0<\varepsilon<\underline{\underline{\mathrm{x}}}-\underline{\mathrm{a}}$ hence $\underline{b}<\frac{x}{\underline{a}+\varepsilon}<\bar{b}$. Thus we obtain that

$$
\left.x=(\underline{a}+\varepsilon) \cdot \frac{x}{\underline{a}+\varepsilon} \in\right] \underline{a}, \bar{a}[\cdot] \underline{b}, \bar{b}[.
$$

b. If $x \in] \underline{a} \bar{b}, \bar{a} \bar{b}[$, then there exists an $\varepsilon>0$ such that

$$
\varepsilon<\overline{\mathrm{b}}-\frac{\mathrm{x}}{\overline{\mathrm{a}}} \text { and } \varepsilon<\overline{\mathrm{b}}-\underline{\mathrm{b}} .
$$


Since $0<\varepsilon<\bar{b}-\frac{x}{\bar{a}}$ we have that $\underline{a}<\frac{x}{\bar{b}-\varepsilon}<\bar{a}$ and since $0<\varepsilon<\bar{b}-\underline{b}$ we have that $\underline{\mathrm{b}}<\overline{\mathrm{b}}-\varepsilon<\overline{\mathrm{b}}$. Thus we obtain that

$$
\left.x=\frac{x}{\bar{b}-\varepsilon} \cdot(\bar{b}-\varepsilon) \in\right] \underline{a}, \bar{a}[\cdot] \underline{b}, \bar{b}[.
$$

As an immediate consequence of Propositions 4., 5. and 6. we can state that

Theorem $\mathbf{3}$ If $0<\mathbf{a}$ and $0<\mathbf{b}$, then $\mathbf{a} \cdot \mathbf{b}=] \underline{a b}, \bar{a} \bar{b}[$.

First, we investigate the case, when the point 0 is an interior point neither of the interval a nor of the interval $\mathbf{b}$.

Theorem 4 1. If $0<\mathbf{a}$ and $0<\mathbf{b}$, then $\mathbf{a} \cdot \mathbf{b}=] \underline{a b}, \bar{a} \bar{b}[$.

2. If $\mathbf{a}<0$ and $\mathbf{b}<0$, then $\mathbf{a} \cdot \mathbf{b}=] \overline{\mathrm{a}} \overline{\mathrm{b}}, \underline{\mathrm{ab}}[$.

3. If $\mathbf{a}<0$ and $0<\mathbf{b}$, then $\mathbf{a} \cdot \mathbf{b}=] \underline{\mathrm{a}} \overline{\mathrm{b}}, \overline{\mathrm{a}} \underline{\mathrm{b}}[$.

4. If $\mathbf{b}<0$ and $0<\mathbf{a}$, then $\mathbf{a} \cdot \mathbf{b}=] \overline{\mathrm{a}} \underline{\mathrm{b}}, \underline{\mathrm{a}} \overline{\mathrm{b}}[$.

The following figures illustrate some cases of the Theorem.
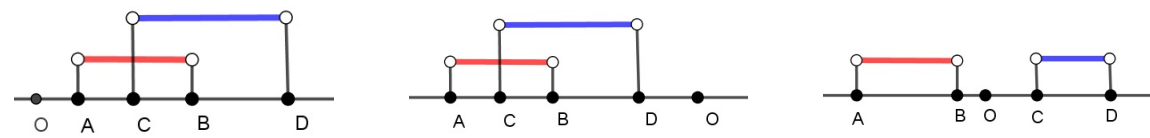

\section{Proof.}

1. Is evident by Theorem 3 .

2. By Proposition 4. and by assertion 1 . we obtain that

$$
] \underline{a}, \bar{a}[\cdot] \underline{b}, \bar{b}[=(-1)(-1)]-\bar{a},-\underline{a}[\cdot]-\bar{b},-\underline{b}[=] \bar{a} \bar{b}, \underline{a b}[.
$$

3. By Proposition 4. and by assertion 1. we obtain that

$$
] \underline{a}, \bar{a}[\cdot] \underline{b}, \bar{b}[=(-1)]-\bar{a},-\underline{a}[\cdot] \underline{b}, \bar{b}[=(-1)]-\bar{a} \underline{b},-\underline{a} \bar{b}[=] \underline{a} \bar{b}, \bar{a} \underline{b}[\cdot
$$

4. Is an immediate consequence of assertion 3 .

Now we investigate the case, when the point 0 is an interior point either of the interval $\mathbf{a}$ or of the $\mathbf{b}$. 
Theorem 5 1. If $0 \in \mathbf{a}$ and $0<\mathbf{b}$, then $\mathbf{a} \cdot \mathbf{b}=] \underline{a b}, \bar{a} \bar{b}[$.

2. If $0 \in \mathbf{a}$ and $\mathbf{b}<0$, then $\mathbf{a} \cdot \mathbf{b}=] \overline{\mathrm{a}} \underline{\mathbf{b}}, \underline{\mathrm{ab}}[$.

3. If $0 \in \mathbf{b}$ and $0<\mathbf{a}$, then $\mathbf{a} \cdot \mathbf{b}=] \underline{\mathrm{ab}}, \overline{\mathrm{a}} \overline{\mathrm{b}}[$.

4. If $0 \in \mathbf{b}$ and $\mathbf{a}<0$, then $\mathbf{a} \cdot \mathbf{b}=] \underline{a} \bar{b}, \underline{a b}[$.

The following figures illustrate some cases of the Theorem.
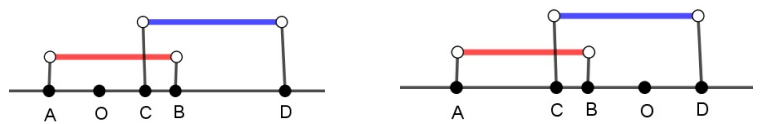

\section{Proof.}

1. By assertions 2. and 1. of Theorem 4. we obtain that

$$
\begin{aligned}
] \underline{\mathrm{a}}, \overline{\mathrm{a}}[\cdot] \underline{\mathrm{b}}, \overline{\mathrm{b}}[= & (] \underline{\mathrm{a}}, 0[\cup\{0\} \cup] 0, \overline{\mathrm{a}}[) \cdot] \underline{\mathrm{b}}, \overline{\mathrm{b}}[= \\
& ] \underline{\mathrm{a}}, 0[\cdot] \underline{\mathrm{b}}, \overline{\mathrm{b}}[\cup\{0\} \cup] 0, \overline{\mathrm{a}}[\cdot] \underline{\mathrm{b}}, \overline{\mathrm{b}}[= \\
& ] \underline{\mathrm{a}} \overline{\mathrm{b}}, 0[\cup\{0\} \cup] 0, \overline{\mathrm{a}} \overline{\mathrm{b}}[= \\
& ] \underline{\mathrm{a}} \overline{\mathrm{b}}, \overline{\mathrm{a}} \overline{\mathrm{b}}[\mathrm{S}
\end{aligned}
$$

2. By assertions 2. and 4. of Theorem 4. we obtain that

$$
\begin{aligned}
] \underline{\mathrm{a}}, \overline{\mathrm{a}}[\cdot] \underline{\mathrm{b}}, \overline{\mathrm{b}}[= & (] \underline{\mathrm{a}}, \overline{\mathrm{a}}[\cdot \cup\{0\} \cup] 0, \overline{\mathrm{a}}[) \cdot] \underline{\mathrm{b}}, \overline{\mathrm{b}}[= \\
& (] \underline{\mathrm{a}}, 0[\cdot] \underline{\mathrm{b}}, \overline{\mathrm{b}}[) \cup\{0\} \cup(] 0, \overline{\mathrm{a}}[\cdot] \underline{\mathrm{b}}, \overline{\mathrm{b}}[)= \\
& ] 0, \underline{\mathrm{ab}}[\cup\{0\} \cup] \overline{\mathrm{a}} \underline{\mathrm{b}}, 0[= \\
& ] \overline{\mathrm{a}} \underline{\mathrm{b}}, \underline{\mathrm{ab}}[.
\end{aligned}
$$

3. Can be obtained from assertion 1 . by changing the roles of a and $b$.

4. Can be obtained from assertion 2 . by changing the roles of a and $b$.

Finally, we investigate the case, when the point 0 is an interior point both of $] \underline{\mathrm{a}}, \overline{\mathrm{a}}[$ and $] \underline{\mathrm{b}}, \overline{\mathrm{b}}[$.

Theorem 6 If $0 \in \mathbf{a} \cap \mathbf{b}$, then $\mathbf{a} \cdot \mathbf{b}=] \min \{\underline{a} \bar{b}, \bar{a} \underline{b}\}, \max \{\underline{a b}, \bar{a} \bar{b}\}[$.

The following figure illustrates the Theorem.

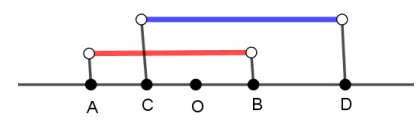


Proof. By Theorem 5. we obtain that

$$
\begin{aligned}
] \underline{\mathrm{a}}, \overline{\mathrm{a}}[\cdot] \underline{\mathrm{b}}, \overline{\mathrm{b}}[= & ] \underline{\mathrm{a}}, \overline{\mathrm{a}}[\cdot(] \underline{\mathrm{b}}, 0[\cup\{0\} \cup] 0, \overline{\mathrm{b}}[)= \\
& \underline{\mathrm{a}}, \overline{\mathrm{a}}[\cdot] \underline{\mathrm{b}}, 0[\cup\{0\} \cup] \underline{\mathrm{a}}, \overline{\mathrm{a}}[\cdot] 0, \overline{\mathrm{b}}[= \\
& ] \overline{\mathrm{a}} \underline{\mathrm{b}}, \underline{\mathrm{ab}}[\cup\{0\} \cup] \underline{\mathrm{a}} \overline{\mathrm{b}}, \overline{\mathrm{a}} \overline{\mathrm{b}}[= \\
& ] \min \{\underline{\mathrm{a}} \overline{\mathrm{b}}, \overline{\mathrm{a}} \underline{\mathrm{b}}\}, \max \{\underline{\mathrm{ab}}, \overline{\mathrm{a}} \overline{\mathrm{b}}\}[.
\end{aligned}
$$

Example 1 Let $\mathbb{F}=\mathbb{Q}(\sqrt{2})$ equipped with the usuall field operations and order. Let $\mathbf{a}:=]-1-\sqrt{2}, 1+2 \sqrt{2}], \mathbf{b}:=[1-2 \sqrt{2}, 2-\sqrt{2}]$. Calculate the product $\mathbf{a} \cdot \mathbf{b}$. Since

$$
\begin{aligned}
& \underline{\mathrm{a}} \cdot \overline{\mathrm{b}}=-\sqrt{2}=-1.4142 \ldots \quad \text { and } \quad \overline{\mathrm{a}} \cdot \underline{\mathrm{b}}=-7 \\
& \underline{a} \cdot \underline{b}=3+2 \sqrt{2}=4.4142 \ldots \quad \text { and } \quad \bar{a} \cdot \bar{b}=-2+3 \sqrt{2}=2.2426 \ldots \text {, }
\end{aligned}
$$

thus by Theorem 6. we obtain that

$$
\mathbf{a} \cdot \mathbf{b}=[-7,3+2 \sqrt{2}[.
$$

Problem 1 Check equation (4) for any ordered field $\mathbb{F}=\mathbb{F}(+, \cdot, \leqslant)$.

\section{References}

[1] M. J. Cloud, R. B. Kearfott, R. E. Moore, Introduction to Interval Analysis, Philadelphia: Society for Industrial and Applied Mathematics (SIAM).

[2] E. Davis, Constraint propagation with interval labels . Artifical Intelligence, 32(3) (1987) 281-331.

[3] Á. Figula, Á. Száz, Graphical relationships between the infimum and intersection convolutions. Math. Pannonica, 21/1 (2010) 23-35.

[4] L. Fuchs, Partially Ordered Algebraic Systems, Dover Publications, Inc. Minesota, New York. 1963.

[5] R. J. Hanson, Interval arithmetic as a closed arithmetic system on a computer. Technical Memorandum 197, Jet Propulsion Laboratory, Section 314, California Institute of Technology, Pasadena, CA. 
[6] T. Glavosits, Zs. Karácsony, Sums and products of intervals in ordered semigroups, An. St. Univ. Ovidius Constanta, Vol. 29 (2) (2021), 187198.

[7] T. Glavosits, Zs. Karácsony, Existence and unicity theorems for additive and logarithmic functional equations, accepted in Communications in Mathematics, 2021.

[8] T. Glavosits, Á. Száz, On the existence of nonnegativity domains of subsets of groups. Demonstratio Math., 37 (2004), 505-516.

[9] L. Jaulin, M. Kieffer, O. Didrit, É. Walter, Applied Interval Analysis. Springer, London, (2001).

[10] W. Kahan, A more complete interval arithmetic, Lecture notes for a summer course. University of Toronto, Canada

[11] R. B. Kearfott, Rigorous Global Search: Continuous Problems, Kluwer, Dordrecht, (1996)

[12] F. W. Levi, Arithmetische Gesetze im Gebiete diskreter Gruppen, Rend. Circ. Mat. Palermo, 35 (1913), 225-236.

[13] P. Lorenzen, Abstrakte Begründung der multiplikativen Idelatheorie, Math. Z., 45 (1939), 533-553.

[14] R. E. Moore, Automatic error analysis in digital computation. Technical Report LMSD-48421 Lockheed Missiles and Space Co, Palo Alto, CA., (1959)

[15] R. E. Moore, Interval Arithmetic and Automatic Error Analysis in Digital Computing. Department Analysis, Stanford University, (1962)

[16] R. E. Moore, Interval Analysis. Englewood Cliff, New Jersey, USA: Prentice-Hall., 35 (1966)

[17] J. J. Moreau, Inf-convolution, sous-additivité, convexité des fonctions numériques. J. Math. Pures Appl., 49 (1970) 109-154.

[18] H. Simbireva, On the theory of partially ordered groups, Mat. Sb., 20 (1947), 145-178. (in Russian) 